\title{
Novel concept for three-dimensional polymer waveguides for optical on-chip interconnects
}

\author{
M. Schröder \\ schroeder@bias.de \\ M. Bülters \\ C. von Kopylow
}

\section{R. B. Bergmann}

BIAS, Bremer Institut für angewandte Strahltechnik, Klagenfurter Straße 2, 28359 Bremen, Germany

BIAS, Bremer Institut für angewandte Strahltechnik, Klagenfurter Straße 2, 28359 Bremen, Germany

BIAS, Bremer Institut für angewandte Strahltechnik, Klagenfurter Straße 2, 28359 Bremen, Germany

BIAS, Bremer Institut für angewandte Strahltechnik, Klagenfurter Straße 2, 28359 Bremen, Germany

We present a simulation study and first experimental implementations for a novel polymer three-dimensional waveguide design. The structures described here allow for new concepts of on-chip communication. By using direct laser writing, free-formed polymer structures can be realized directly on the surface of integrated circuits on wafer- or die-level. Further photonic structures like waveguides, resonators, splitters and couplers can be realized with an extended freedom of design to the third dimension. Our approach opens new possibilities for optical interconnects and routing for on-chip signal transmission with a high fill factor and CMOS compatibility.

[DOl: http://dx.doi.org/10.2971/jeos.2012.12027]

Keywords: polymer optical waveguide, 3D-structures, direct laser writing, on-chip optical routing

\section{INTRODUCTION}

This paper presents a new concept of optical on-chip interconnects and its technology. Two-photon lithography enables the fabrication of three-dimensional micro and nanostructures in photosensitive materials. Hence new design potentials of waveguiding structures with new functions and a high fill factor are at hand.

Increasing demands on the data rate in integrated circuits (IC) require integration with high fill factors and low energy consumption. High data rates and routing between different nodes or cores on one chip are of great interest [1]. Optical links and components fulfil these requirements and are currently available [2].

For photonic integrated circuits silica, silicon, III-V semiconductors and polymers are the most popular materials. Many polymer materials have a broad transparency range and good processing characteristics by lithographic development; however, their refractive index is rather low compared to semiconductors. Thus polymer waveguides on semiconductors can just be realized with additional technical effort, for example using $\mathrm{SiO}_{2}$ insulator layers [3]. Modern lithographic technologies, like direct laser writing based on the two-photon polymerization, allow improvements in this aspect. Threedimensional structuring of photonic devices directly on the IC interface at wafer- and die-level is feasible [4]. The threedimensional structuring of polymers in the sub-micrometer range, allows for a new approach of waveguide profile design, with additional degrees of freedom compared to conventional waveguides, which are integrated by planar technology.
Planar techniques like SOI (silicon on isolator) are commonly used to realise optical connections at wafer level. Therewith adjacent optical waveguides with high-frequency signals induce less crosstalk at high integration density and offer further advantages such as electromagnetic compatibility and nearly lossless transmission. However the light propagation occurs just in one or few discrete planes. Therefore, the integration of the optical interconnects is implemented by extensive technological effort like flip-chip mounting [5]. Due to the constrained design rules the devices have a low fill factor and a limited IC interface layout. Moreover techniques for the integration of photonic layers have to show CMOS technology compatibility.

In classic ridge waveguide simulation, the beam propagation method (BPM) is commonly used. There are many different algorithms [6] and commercial software solutions available. However the classic BPM is an approximation technique for slowly varying optical waveguides without time dependency. For three-dimensional structures the finite element BPM [7] had been introduced.

Free formed models can also be solved by rigorous methods. Here we present the numerical simulation of complex three-dimensional waveguide structures with finite integration technique (FIT) by CST Microwave Studio [8]. In this approach the model is divided by a hexagonal mesh with mesh cells one order smaller than the simulated wavelength. For the exact calculation of Maxwell's equations in every cell, the computing time gets very high. 

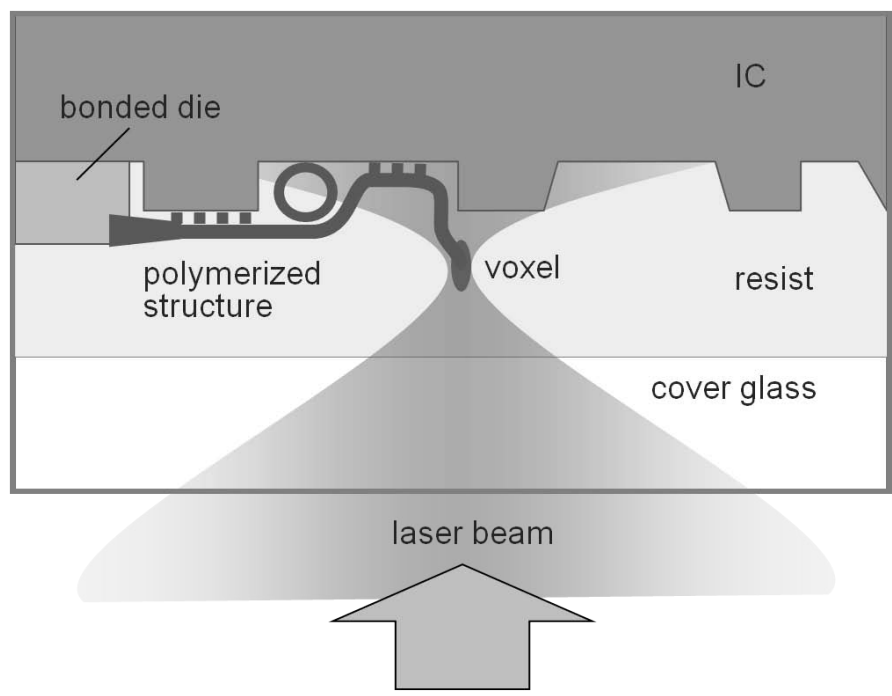

FIC. 1 Principle of waveguide fabrication on IC surface via 3D laser lithography: The IC surface is mounted upside down in a sandwich structure on a cover glass and the space is filled with resist. A laser beam with a wavelength of $780 \mathrm{~nm}$ and a $70 \mathrm{fs}$ pulse width is focussed by an inverse microscope into the resist. Polymerization occurs only at the focal point (voxel) by two-photon absorption.

\section{MATERIALS AND EXPERIMENTAL METHODS}

For the experimental realization of the designed 3D structures, we use the laser lithography system Photonic Professional [9]. During the writing process, a pulsed laser beam of $780 \mathrm{~nm}$ wavelength is focused in the UV-sensitive polymer resist. The pulse width is $70 \mathrm{fs}$ with a repetition rate of $100 \mathrm{MHz}$ and an average output power of $65 \mathrm{~mW}$. Many UV-sensitive resists like standard SU-8 can be used, in the following experiments we use IP-L resist (Nanoscribe corporation) [10] which is viscous and needs no prebake. Its refractive index (at $780 \mathrm{~nm}$ ) changes from 1.48 to 1.52 after exposure. After the writing process, the sample is treated for 30 minutes in a developer bath and finally cleaned by isopropanol. The whole process is CMOS-compatible. The resist is developed only in the small focusing volume (called voxel) where intensities are high enough for two photon absorption. By moving the sample using a 3D nanometer precision piezo stage, the minimum size of arbitrary shaped structures is only limited by the voxel size of at least $120 \mathrm{~nm}$ in lateral and $300 \mathrm{~nm}$ in vertical direction [11]. The voxel size is depending on the laser power employed and can be adjusted by an acousto-optic modulator. By a smart arrangement of refractive and diffractive optical elements, a complete optical system can be integrated in a miniaturized form.

Figure 1 shows the fabrication process on an IC surface. In our setup the laser beam is focussed by an inverse microscope through a cover glass into the resist. The IC surface is mounted upside down in a sandwich structure on the cover glass and the space is filled with resist. The interface between IC surface and resist can also be identified via the microscope. By using the physical process of the two-photon absorption, an exposure of a small region in a transparent bulk material is possible [12]. Based on a precise beam alignment and guidance, almost

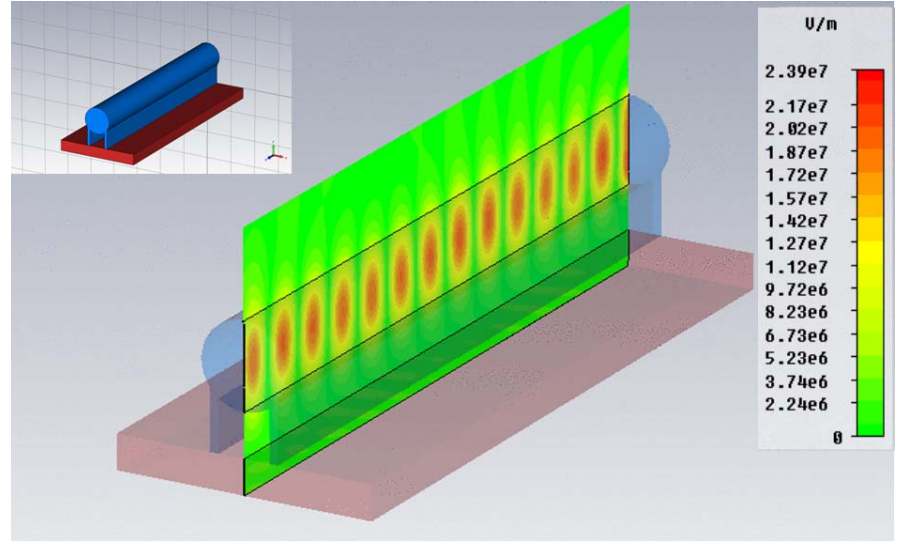

FIG. 2 Simulation of electric field distribution for a round waveguide on bars at $\lambda=1.55 \mu \mathrm{m}$ with a diameter of $1 \mu \mathrm{m}$. The colorscale shows the magnitude of the electric field ranging from o (green, bottom) to $2 \cdot 10^{7} \mathrm{~V} / \mathrm{m}$ (red, top). Inset: geometry of the waveguide (blue) separated from the Si-substrate (red) by two bars (blue).

every three dimensional pattern is realisable with this method which allows structure sizes in the sub-wavelength scale.

The alignment of these optical structures to the finished CMOS devices is determined via alignment marks on the electronic interface. For minor inaccuracies and deviations the laser lithographical system provides offset-, tilt- and scalecorrection functions. Thereby optical interconnects between electro optical links can be built-on in a high efficiently way. Hence tapered structures of the waveguides which operate as an optical funnel, as well as prism or grating coupling can increase the efficiency.

Due to the use of a broadband transparent polymer material as for example SU-8 or IP-L resist, the usage of the wavelength becomes more flexible [13]. Wavelengths below the silicon band gap of $1100 \mathrm{~nm}$ enable the direct integration of the photodiode in the IC-Chip environment by a $\mathrm{p}(\mathrm{i}) \mathrm{n}$ junction doping profile.

\section{SIMULATION OF LIGHT PROPAGATION IN WAVEGUIDES}

To realize 3D waveguides on IC surfaces with a nearly lossless propagation, the waveguide has to ensure a total reflection at the interface to the surrounding material. For this purpose the refractive index of the core must be higher than the refractive index of the cladding. The waveguides, typically composed of polymer materials with a refractive index about 1.5 , are enveloped in air or vacuum with a refractive index of about 1 . Our approach for the realization of polymer waveguides on silicon is a spatial separation by an air gap to avoid the need for an additional $\mathrm{SiO}_{2}$ layer and to preserve the high index contrast in all directions. Hence the optical structures are not limited to one plane and can use the whole space above the substrate.

Figure 2 shows a waveguide with a circular cross section, as an obvious simple design, positioned on two parallel aligned bars of the same material. The round shape of the waveguide leads to the same transmission mode for TE and TM polariza- 


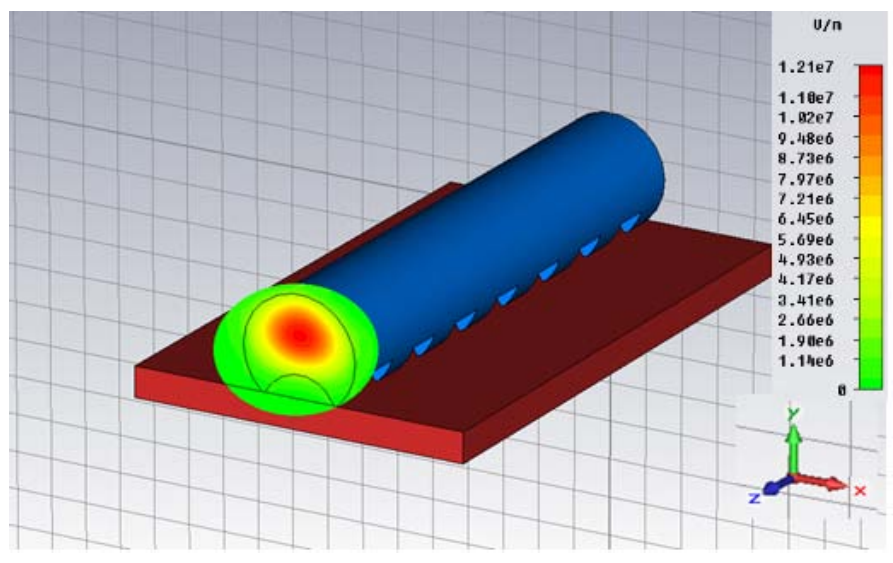

FIG. 3 Principle design of a kidney-shaped waveguide (blue) on Si-substrate (red). The inset colorscale shows the electric field distribution at $\lambda=1.55 \mu \mathrm{m}$ ranging from 0 (green, bottom) to $10^{7} \mathrm{~V} / \mathrm{m}$ (red, top). The two lines of periodically arranged pillars separate the waveguide from the substrate.

tion, which is an advantage for three-dimensional bends. For the optimization of the transmission there are various adjustment parameters of the structures. The essential ones are: The diameter, the distance to the substrate and the dimensions of the bars. The bars should minimize loss, thus sub-wavelength dimensions are required, but need to be still stable enough for a firm construction.

By simulation we investigate the crosstalk of two waveguides with a round shape, a diameter of $1.4 \mu \mathrm{m}$ and a length of $50 \mu \mathrm{m}$. At a distance of $4 \mu \mathrm{m}$ between two parallel aligned waveguides, we find an overall transmission of less than $1 \%$ at $\lambda=1.55 \mu \mathrm{m}$. For two waveguides crossing orthogonal to each other at a distance of $4 \mu \mathrm{m}$, we find a transmission of $0.02 \%$ for orthogonal crosses, so a high integration density is feasible.

Figure 3 shows a kidney-shaped waveguide. An advantage of this structure is the higher mechanical stability and better adhesion due to periodic pillars separating the waveguide from the substrate. The simulated mode of the structure with a width of $3 \mu \mathrm{m}$ is shown in Figure 3 as well. The E-field distribution of the horizontal polarized $\mathrm{TE}_{00}\left(\mathrm{E}^{\mathrm{x}}\right)$ mode is mapped at the end face of the waveguide. A Gaussian distribution with a slightly elliptical profile results with a FWHM (full width at half maximum) of $2.0 \mu \mathrm{m}$ in latitude and $1.5 \mu \mathrm{m}$ in altitude at a maximum diameter of $3 \mu \mathrm{m}$.

\section{EXPERIMENTAL RESULTS}

In order to show the feasibility of 3D laser lithography we fabricate test structures that demonstrate the concept of the waveguide design simulated in the previous chapter.

\subsection{Realization of polymer structures on a glass substrate}

Figure 4 shows a scanning electron microscope (SEM) image of first experimental implementations of polymer structures realized by direct laser writing. The structure is fabricated using IP-L resist on a glass substrate. In the previous chapter

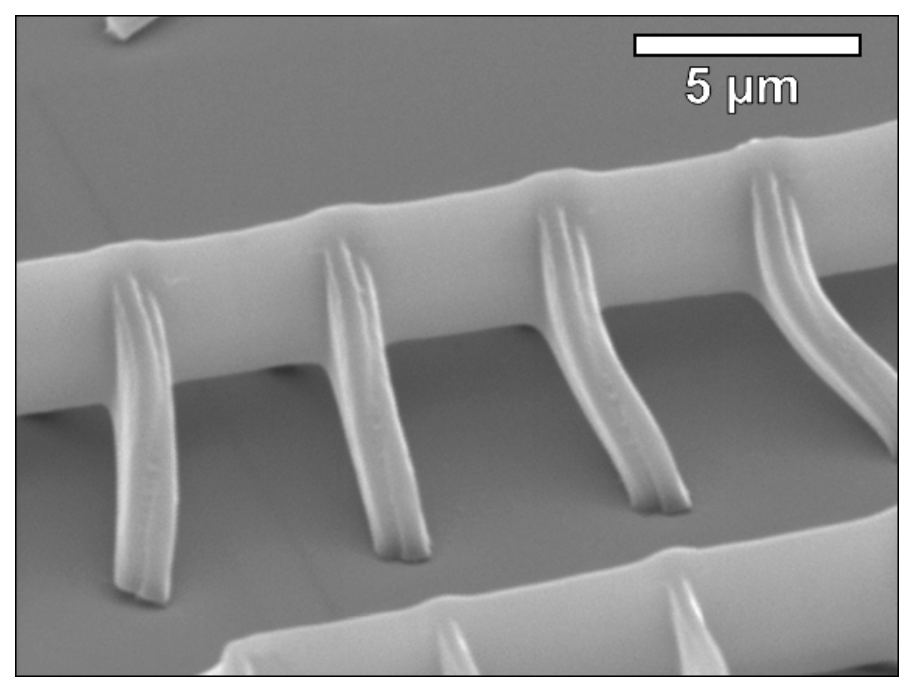

FIG. 4 Polymer structure on stilts with an air gap to the glass surface (SEM image).

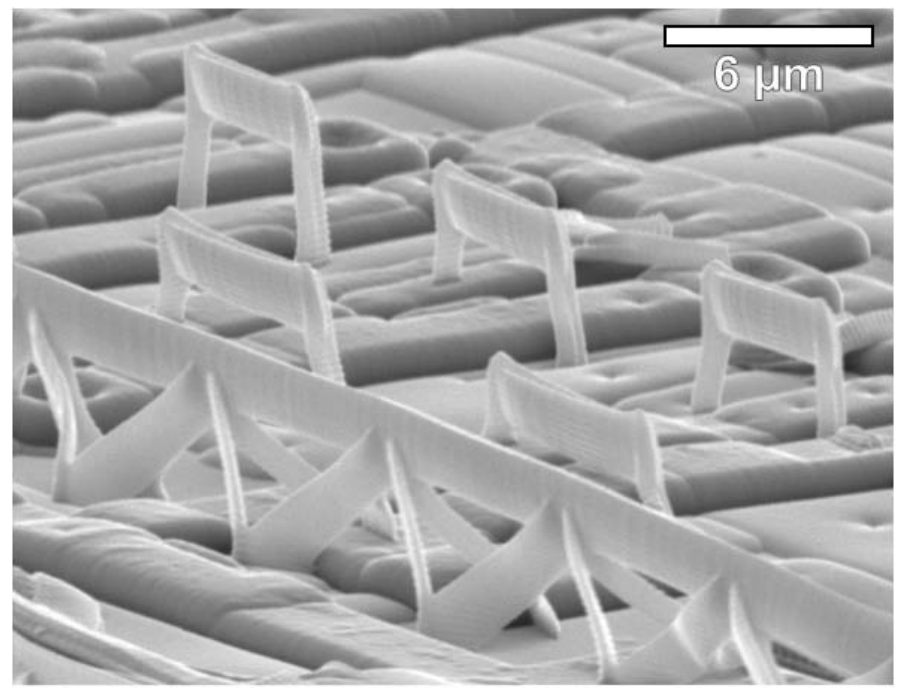

FIG. 5 Three-dimensional polymer structure on stilts integrated directly on an IC surface (SEM image).

we simulated a waveguide on continuous bars to form a gap to the substrate and we showed the feasibility of more complex shaped waveguides with periodic pillars, see Figure 3. In the structure shown in Figure 4, the air gap to the substrate is formed by periodic stilts with a distance of $4 \mu \mathrm{m}$.

\subsection{Realization of polymer structures on IC surface}

Figure 5 shows first experimental implementations of 3D polymer structures on an IC surface. In the experiment a fully processed wafer is introduced in the lithography system as described in Figure 1. The SEM image shows the surface of the metallic wires of an integrated circuit. The height deviation of the topography is about $1 \mu \mathrm{m}$. The polymer structure is based on stilts with an air gap of $3 \mu \mathrm{m}$ to the substrate.

\section{CONCLUSION}

We demonstrate simulation studies based on finite integration technique of three-dimensional photonic structures with 
novel profile designs, which have been implemented by 3D laser lithography. Employment of three-dimensional structures is an effective way to use the free space above the wafer surface for instance with polymer waveguides on stilts. First experimental implementations of polymer structures on IC surface are presented. The realization of optical waveguides on an IC surface therefore appears feasible using threedimensional design freedom. Further work will concern the integration of more complex structures, such as the waveguides simulated in this paper, as well as optical characterization of the fabricated structures. With this novel concept a very flexible, efficient and straightforward coupling of onchip links, fibres and dies appears possible.

\section{References}

[1] D. A. B. Miller, "Optical interconnects to electronic chips," Appl. Optics 49, 59-70 (2010).

[2] A. Biberman, B. Lee, N. Sherwood-Droz, M. Lipson, and K. Bergman, "Broadband Operation of Nanophotonic Router for Silicon Photonic Networks-on-Chip," IEEE Photonic. Tech. L. 22, 926-928 (2010).

[3] B. Yang, L. Yang, R. Hu, Z. Sheng, D. Dai, Q. Liu, and S. He, "Fabrication and Characterization of Small Optical Ridge Waveguides Based on SU-8 Polymer," J. Lightwave Technol. 27, 4091-4096 (2009).

[4] T. Grossmann, S. Schleede, M. Hauser, T. Beck, M. Thiel, G. von Freymann, T. Mappes, and H. Kalt, "Direct laser writing for active and passive high-Q polymer microdisks on silicon," Opt. Express 19, 11451-11456 (2011).
[5] A. Shacham, K. Bergman, and L. Carloni, "Photonic Networks-onChip for Future Generations of Chip Multiprocessors," IEEE T. Comput. 57, 1246-1260 (2008).

[6] H.-P. Nolting, and R. März, "Results of Benchmark Tests for Different Numerical BPM Algorithms," J. Lightwave Technol. 13, 216-224 (1995).

[7] Y. Tsuji, M. Koshiba, and T. Shiraishi, "Finite Element Beam Propagation Method for Three-Dimensional Optical Waveguide Structures," J. Lightwave Technol. 15, 1728-1734 (1997).

[8] I. Munteanu, M. Timm, and T. Weiland, "It's About Time," IEEE Microw. Mag. 11, 60-69 (2010).

[9] N. Anscombe, "Direct laser writing," Nat. Photonics 4, 22-23 (2010).

[10] M. Thiel, J. Fischer, G. von Freymann, and M. Wegener, “Direct laser writing of three-dimensional submicron structures using a continuous-wave laser at 532 nm," Appl. Phys. Lett. 97, 221102 (2010).

[11] M. Deubel, G. von Freymann, M. Wegener, S. Pereira, K. Busch, and C. M. Soukoulis, "Direct laser writing of three-dimensional photonic-crystal templates for telecommunications," Nat. Mater. 3, 444-447 (2004).

[12] K. Busch, G. von Freymann, S. Linden, S. F. Mingaleev, L. Tkeshelashvili, and M. Wegener, "Periodic nanostructures for photonics," Phys. Rep. 444, 101-202 (2007).

[13] A. Borreman, S. Musa, A. Kok, M. Diemeer, and A. Driessen, "Fabrication of Polymeric Multimode Waveguides and Devices in SU-8 Photoresist Using Selective Polymerization," in IEEE/LEOS Benelux Chapter 2002 Annual Symposium 83-86 (Vrije Universiteit Amsterdam, Amsterdam, 2002). 